\title{
Posterior meniscal root repair: a biomechanical comparison between human and porcine menisci
}

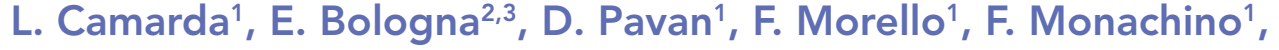 \\ F. Giacco ${ }^{4}$, M. Zingales ${ }^{2,3}$
}

1 Department of Orthopaedic Surgery, University of Palermo (DiChirOnS), Palermo, Italy

2 Dipartimento di Ingegneria Civile, Ambientale, Aerospaziale, dei Materiali (DICAM), Palermo, Italy

3 Bio/NanoMechanics for Medical Sciences Laboratory, Advanced Technology Network (ATeN)-Center, Palermo, Italy

${ }^{4}$ Department of Orthopaedic Surgery, IRCCS Istituto Ortopedico Galeazzi, Milano, Italy

\section{CORRESPONDING AUTHOR \\ Lawrence Camarda \\ Department of Orthopaedic Surgery (DiChirOnS) \\ University of Palermo \\ Via del Vespro, Palermo, Italy \\ Phone: +390916554140 \\ Fax: +39091655 4111 \\ E-mail: lawrence.camarda@unipa.it}

DOI

10.32098/mltj.01.2019.03

\begin{abstract}
SUMMARY
Background. The aim of the present study was to compare the biomechanical characteristics of posterior meniscal root repair of porcine and human menisci. Methods. In vitro biomechanical testing was performed using 12 porcine menisci and 12 human menisci. All menisci were sectioned at the midpoint of their circumference and mounted on an electro-mechanic testing machine. The posterior root was sutured with three single stitches using a no. 2 non-absorbable suture. All specimens were subjected to cyclic axial loading followed by load-to-failure testing. Displacements were recorded at the conclusion of cycles 1, 100, 500 and 1000. Further, load-displacement curves of each specimen were recorded and analyzed to determine the cyclic stiffness at the 500th and 1000th cycle. Results. A higher elongation of the human specimens was observed after 1,100,500 and 1000 loading cycles, when it was compared to porcine specimens $(\mathrm{p}<0.05)$. The total displacement amount of the porcine specimens at the 1000th cycle was $2.2 \mathrm{~mm} \pm 0.1$. Similarly, the total displacement amount of the human specimens at the 1000th cycle was $3 \mathrm{~mm} \pm 0.5$. Higher stiffness was observed in the porcine group at the 500th and 1000th cycle. Conclusion. Three single sutures technique for meniscal root repair appears to be biomechanically effective for both human and porcine menisci.
\end{abstract}

\section{KEYWORDS}

meniscal root repair; meniscal root fixation; meniscal root fixation; suture menisci; porcine menisci; buman meniscal root

\section{INTRODUCTION}

Meniscal root repair (MRR) techniques have recently gained popularity, especially following an increased knowledge of meniscal root on knee biomechanics. In fact, previous studies have suggested that the integrity of posterior meniscal root is essential for maintaining circumferential meniscal hoop tension $(1,2)$. In cases of posterior root injuries, the transmission of the circumferential hoop tension is impaired, and the menisci tend to be extruded and displaced anteriorly and posteriorly. This can lead to increased tibiofemoral contact pressures that have been reported to be equivalent to a complete meniscectomy (3). These changes are responsible for accelerated degenerative changes within the knee joint. Recently, the effectiveness of meniscal root repair has been demonstrated to restore the loading profiles to the intact knee, for both medial and lateral meniscus (3-6). The key of an effectiveness MRR is represented by an anatomical repair using a consistent root reattachment, using several surgical techniques with different methods of suturing the meniscal root. Previous studies have demonstrated that the number and size of sutures used during MRR could have a role to obtain a suboptimal construct, resisting to elongation during biomechanical tests (7-8). In addition, three simple stitches have been effective during MRR, reducing displacement of the posterior medial meniscal root in response to cyclic loading after transtibial pull-out repair (7). Whereas human menisci have been characterised relatively well in several studies, mostly MRR biomechanical studies were 
performed using a porcine model, with the main advantage of standardized tissue and bone quality. This has offered a base for comparative studies on MRR present in the current biomechanical literature. On the contrary, human menisci present an extreme variability, mainly linked to the age, sex, and degree of joint degeneration. Further, in recent studies a high number of middle-aged patients treated for medial posterior MRR was observed, and often the patients were over 50 years of age $(9,10)$. In these patients, degenerative meniscal changes could be expected, altering the corresponding treatment of the meniscal root tears. Even if it has been found that in such circumstances meniscal biomechanics behaviour could be compromised, no data is available concerning meniscal root repair in an already degenerative meniscus. Further, in these cases applying three simple stitches during meniscal root repair might be beneficial for healing of the posterior meniscal root, potentially reducing the post-operative immobilization time.

The aim of the present study was to compare the biomechanical characteristics of posterior MRR of porcine and human middle-aged menisci using three single sutures technique. We hypothesized that both constructs have similar properties concerning cyclic displacement, stiffness, and ultimate failure load.

\section{MATERIALS AND METHODS}

In vitro biomechanical testing was carried out using 12 porcine menisci and 12 human menisci. Fresh porcine knees were obtained for the experiments from a local butcher, stored at $-20{ }^{\circ} \mathrm{C}$ and then thawed at room temperature 12 $\mathrm{h}$ before use. Skin and subcutaneous tissue were removed around the joint, and the menisci were sharply dissected from the bony attachment to the tibia plateau. Posterior root attachments were sectioned at its posterior attachment, preserving the integrity of the meniscus itself. Similarly, human menisci were collected during operations of total knee replacements (mean patient age of 55.3 years, range 50-70 years). Menisci that presented clear macroscopic degenerative changes such as horizontal and complex tears were excluded from the present study. Meniscal harvesting was performed after proximal tibial cut. During this phase, care was taken to detach the anterior and posterior meniscal root from its bony insertion. The posterior meniscal root was then signed with a dermographic marker, to differentiate it from the anterior root during the subsequent phase of the study. All menisci were then sectioned at the midpoint of their circumference. During preparation and mechanical testing, all menisci were kept moist to prevent drying, being sprayed with saline solution. Each specimen was mounted on an electro-mechanic testing machine (ElectroForce 3330, BOSE) equipped with a $1-\mathrm{kN}$ load cell. The sectioned portion of the menisci was mounted in a custommade clamp directly fixed to the actuator of the tensile testing machine (11). Care was taken in this phase to fix the menisci allowing an axial load in line with the circumferential fibers of the meniscal root. The experimental set up of a generic test is reported in figure $\mathbf{1}(\mathbf{a}, \mathbf{b})$ with details on the clamped side of menisci and the custom-made clamp as well as the surgical sutures.

As per standard intra-operative technique, the meniscal root was sutured with three single stitches using a curved a)

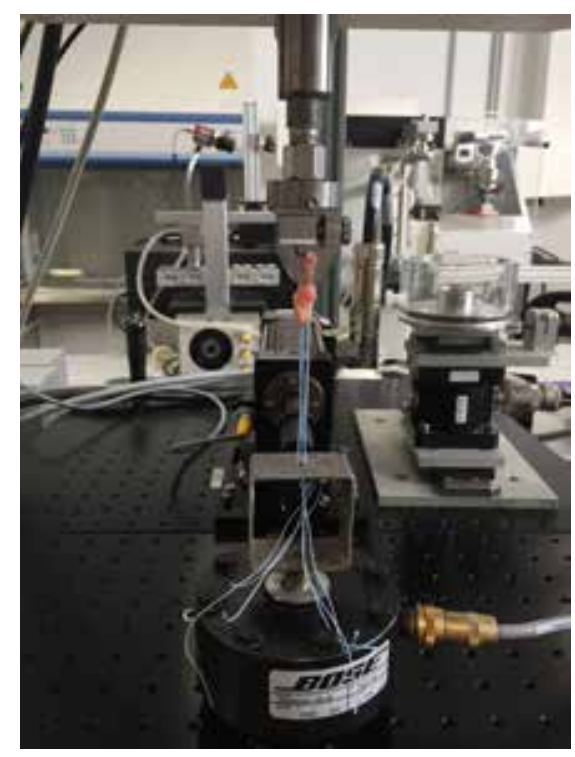

b)

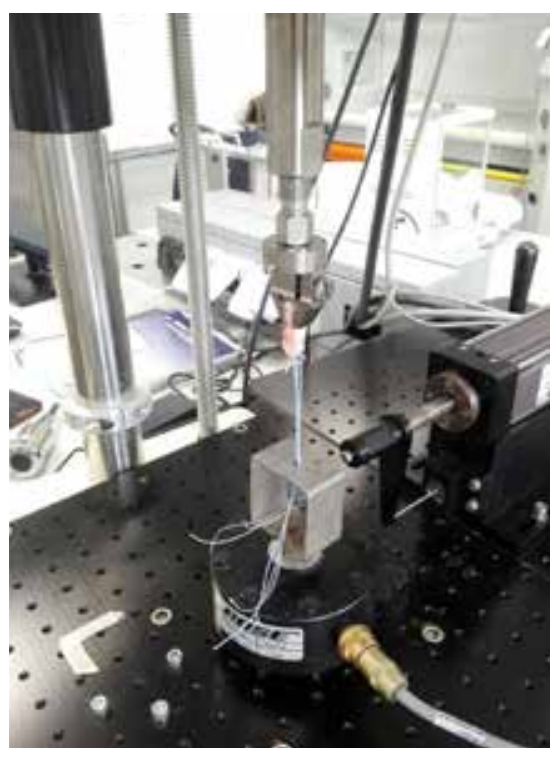

Figure 1 a) Experimental set up of the testing machine and specimen conditions; b) Custom made clamp and surgical suture of menisci specimens. 
tapered needle and a non-absorbable suture (no. 2 Fiberwire, Anthrex, Inc) (figure 1, 2) (7). Specifically, two loops were passed $5 \mathrm{~mm}$ from the posterior root attachment of the medial meniscus. An additional suture was passed through the red-red zone of the meniscus, approximately $7 \mathrm{~mm}$ medial to the edge of the root as described by Kim et al $(7,12)$. At this point, all the free ends of the sutures were directly passed through a $5 \mathrm{~mm}$ hole of a steel plate directly fixed to the base of the test frame. The steel plate has been used in other biomechanical studies as it provided a rigid simulation of the bone cortex (13), allowing standardized methods for the purpose of the study. Suture ends were them tied over a surgical button by a single surgeon, taking care to yield a total suture length of $7 \mathrm{~cm}$, that corresponds to the intratunnel suture length. At this point seven half-stitches thrown in an alternating fashion were applied to secure the meniscus-suture construct to the surgical button.

\section{Biomechanical testing}

A specific loading protocol was defined and used for each specimen of the study that was applied after an initial preload of $5 \mathrm{~N}$ obtained with linear rate of $1 \mathrm{~N} / \mathrm{sec}$ to eliminate initial major lacks and slippages for the evaluation of successive loadings. Such preload provides an initial and reproducible reference condition experimentally observable for each specimen. In more detail the used protocol is composed by a sequence of three phases: i) preconditioning; ii) low-frequency load-control cyclic test; iii) displacement-control monotone test. Specifically, phase i) involves a harmonic load-control test with forces with peaks $1 \mathrm{~N}$ and $30 \mathrm{~N}$ with a frequency of $0.5 \mathrm{~Hz}$. Phase i) is followed by a phase ii) of the test protocol that involves the application of sinusoidal loads with a frequency of $0.5 \mathrm{~Hz}$ and peaks $10 \mathrm{~N}$ and $30 \mathrm{~N}$ for a period $\mathrm{T}=2,000.00 \mathrm{sec}$ (1000 complete load cycles). Phase iii) of the testing protocol involves a monotonic, displacement-control test at a machine crosshead speed of $0.5 \mathrm{~mm} /$ sec, in order to maintain the control of the specimen at the onset of failure. The graphical representation of the testing protocol has been reported in figure 3 , depicting the displacements of the clamp as function of time recorded during the test. This protocol was chosen after a literature survey that aimed to identify the most typical benchmark conditions for this type of analysis $(7,8,13)$. Further, these loading parameters have been used by previous studies as they corresponded to tensile forces that the posterior medial meniscal root may experience under neutral rotation, knee flexion from $30^{\circ}$ to $60^{\circ}$, and $500 \mathrm{~N}$ of tibiofemoral loads. In addition, these parameters could represent the range of motions and partial weight-bearing seen over the course of a typical 6-week post-operative rehabilitation program after meniscal root repair $(13,14)$. The amount of meniscal displacement in response to cyclic loading was obtained from the testing machine crosshead displacement. Specifically, displacements were recorded at the conclusion of 1, 100, 500 and 1000 cycles. Further, load-displacement curves of each specimen were recorded and analyzed to determine the cyclic stiffness of the ensemble of meniscal tissue and surgical suture, at the 500th $\left(\mathrm{K}_{500}\right)$ and 1000th $\left(\mathrm{K}_{1000}\right)$ cycle. Furthermore, ultimate failure load $(F)$ was recorded for each specimen. These load

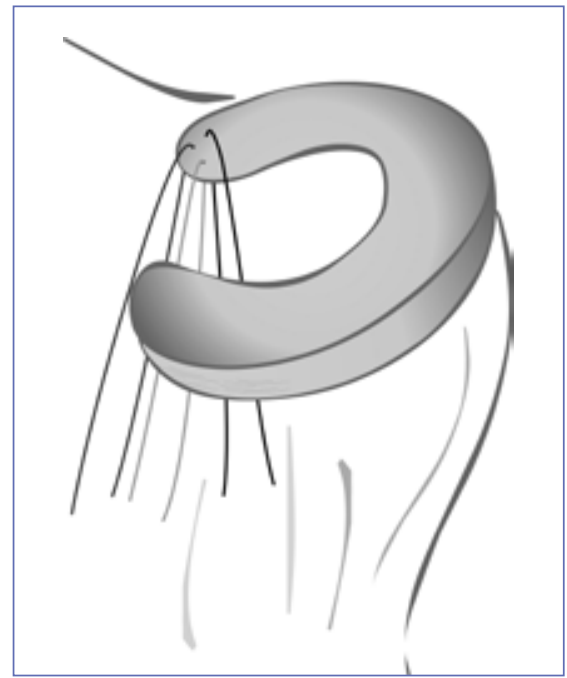

Figure 2 - Three single sutures technique used for the posterior meniscal root fixation.

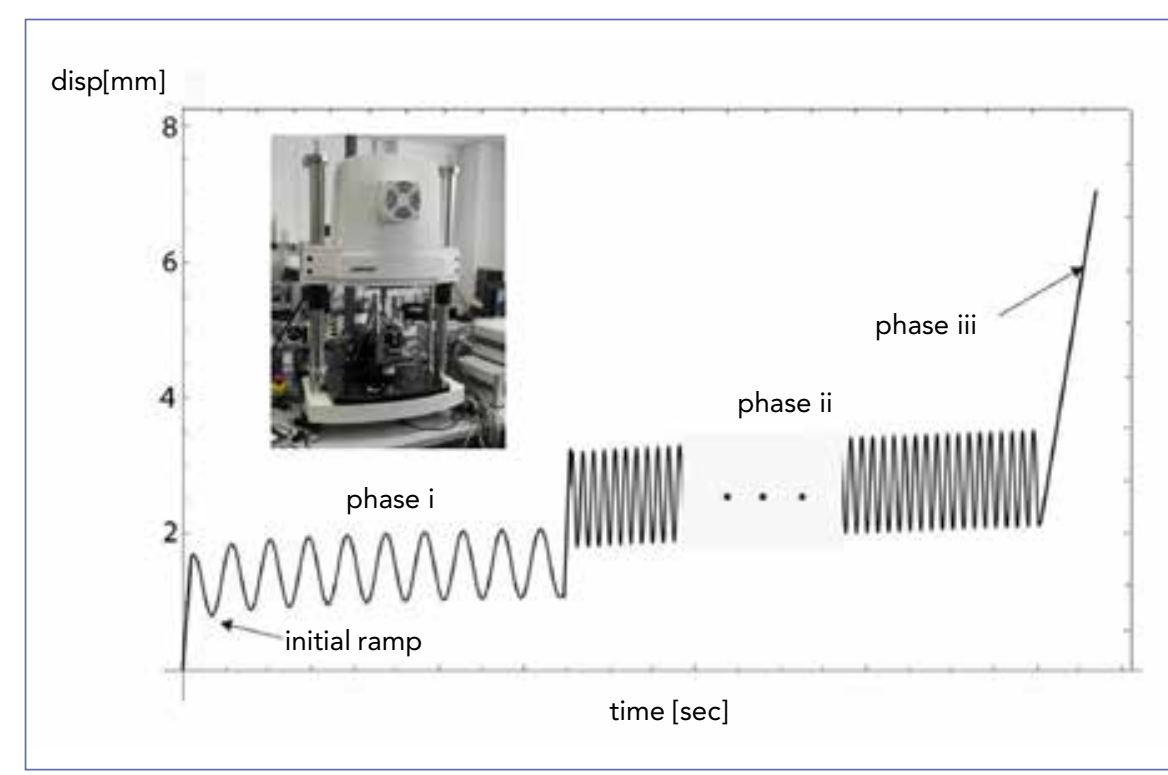

Figure 3 - Illustrative diagram of testing protocol with the three phases. 
conditions allow to assume rigid behavior of load frame and clamps with respect to the investigated meniscal tissue and surgical wire. All research was conducted ethically according to the international standards (15).

\section{Statistical analysis}

Data were analyzed using SPSS statistical software, version 18.0 (SPSS, Inc., Chicago, IL, USA). Means and standard deviation were calculated for each parameter. Tensile properties between two groups were analyzed using Student's t-test with a level of significance at $\alpha=0.05$ and a statistical power of $\beta=0.80$.

\section{RESULTS}

All menisci were successfully tested and survived the phase ii) and iii) of the testing protocol discussed in the previous section. The failure mechanism for all specimens tested was the suture pull-through of meniscal tissue during load to failure tests obtained at the end of phase iii) of the protocol. Concerning the ultimate load-to-failure, significant differences were noted between porcine $\left(F_{\mathrm{p}}=390 \pm 13 \mathrm{~N}\right)$ and human $\left(F_{\mathrm{h}}=161 \pm 25 \mathrm{~N}\right)$ specimens $(\mathrm{p}<0.05)$.

As described in table $\mathbf{I}$, a higher elongation of the human specimens was observed after 1, 100, 500 and 1000 loading cycles, when it was compared to porcine specimens $(\mathrm{p}<$ $0.05)$. The total displacement amount of the porcine specimens at the 1000th cycle was $2.2 \mathrm{~mm} \pm 0.1$. Similarly, the total displacement amount of the human specimens at the 1000th cycle was $3 \mathrm{~mm} \pm 0.5$. The stiffness of the porcine group was of $107 \pm 45 \mathrm{~N} / \mathrm{mm}$ at the 500th cycle and of 115 $\pm 50 \mathrm{~N} / \mathrm{mm}$ at the 1000th cycle. Similarly, the stiffness of the human group was of $77 \pm 18 \mathrm{~N} / \mathrm{mm}$ at the 500th cycle and of $79 \pm 17 \mathrm{~N} / \mathrm{mm}$ at the 1000th cycle (table I). Differences between the two groups were statistically significant. A graphical representation of the average values of the stiffness of the porcine and the human menisci is reported in figure 4 , showing that as the number of cycles increases, an increment in the stiffness of the porcine and the human specimen is observed.

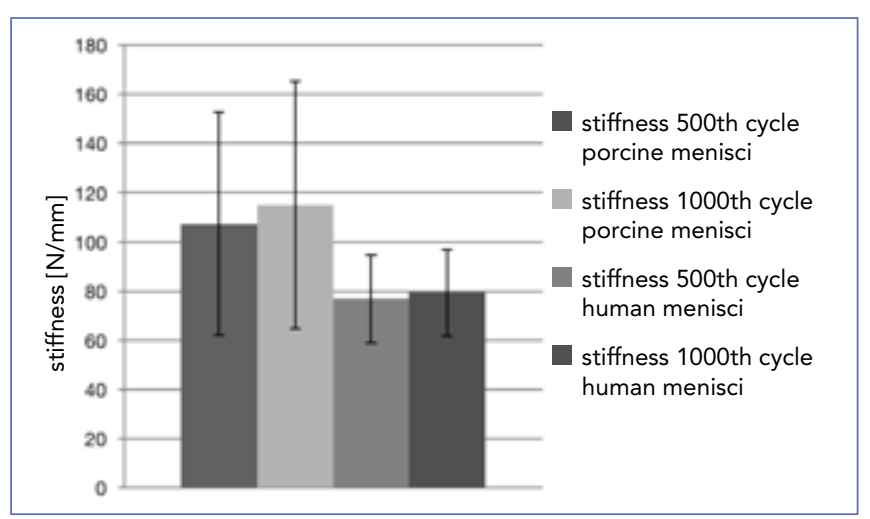

Figure 4 - Comparison among the stiffness of porcine and human specimens for different number of cyclic loads.

\section{DISCUSSION}

The main finding of the present study was that porcine meniscal-suture construct was able to significantly resist displacement compared to human meniscal-suture construct at 1, 100, 500 and 1000 cycles of cyclic tensioning. However, a small amount of displacement under cyclic loads was noted between 2 groups. Further, both constructs appeared to be biomechanically effective, not exceeding $3 \mathrm{~mm}$ of displacement that has been reported to compromise meniscal function.

To date, several biomechanical studies have been conducted to better understand the effectiveness of MRR. Generally, porcine or human menisci have been used obtaining results that differ sensitively making it difficult to compare data. This is because of highly different variables, such as the tissue and sutures used, and different testing machines and testing protocols. In the present study, using the same testing protocol setup authors wanted to analyze biomechanically the posterior MRR, comparing human and porcine menisci using three single stitches. During cyclic loads, porcine specimens presented a total elongation of 22\% (D1-1000 elongation 1-1000 cycles), while human specimens presented an elongation of $34 \%$. Specifically, a whole difference of 0.9 $\mathrm{mm}$ displacement was at the 1000th cycle. Even if this differ-

Table I - Specimens displacement in response to cyclic loading and load to failure.

\begin{tabular}{llllll}
\hline & $\begin{array}{l}\text { displacement } \\
\text { 1st cycle }\end{array}$ & $\begin{array}{l}\text { displacement } \\
\mathbf{1 - 1 0 0} \text { cycle }\end{array}$ & $\begin{array}{l}\text { displacement } \\
\mathbf{1 - 5 0 0} \text { cycle }\end{array}$ & $\begin{array}{l}\text { displacement } \\
\mathbf{1 - 1 0 0 0} \text { cycle }\end{array}$ & ultimate load to failure (N) \\
\hline human specimens & $2.3 \pm 0.5$ & $2.5 \pm 0.5$ & $2.8 \pm 0.5$ & $3.1 \pm 0.5$ & $161.3 \pm 25.5$ \\
\hline porcine specimens & $1.8 \pm 0.1$ & $1.9 \pm 0.1$ & $2.0 \pm 0.1$ & $2.2 \pm 0.1$ & $390 \pm 13.3$ \\
\hline Va
\end{tabular}

Value expressed in millimetres. Data presented as mean \pm standard deviation. $\mathrm{p}<0.05$ (statistically significative difference). 
ence was statistically significant, a real biomechanics effect of this difference in MRR has to be investigated. Further, differences in term of stiffness were noted between groups.

From a biomechanical perspective, the study shows that both constructs undergo a severe time-dependent behavior known as hereditariness. This could be observed in specific fibrous tissue such as soft structural tissues as arteries and blood vessels, as well as in human ligaments and tendons (16-19). The aforementioned behavior may be detected from the observation of figure 3 , which shows that as soon as more cycles are involved, an evident increment in the average value of the displacement is evidenced. In the present study, a highly stiff suture material was used (i.e., Fiberwire, Arthrex Inc), and a solid meniscal clamp. As low loads were applied to the meniscus construct, we believe that the main cause of the progressive and permanent elongation of the constructs could be the slippage of the whole suture material upon the meniscus tissue. This could be influenced by the size and number of sutures used, as different sutures could share loads, potentially reducing slippage effect of meniscal tissue.

To date, there is a lack of consensus regarding the best treatment for patients presenting meniscal root injury. Conservative treatment has been advocated in elderly patients, poor surgical candidates, or patients with advanced arthritis (20). It consists in anti-inflammatory administration, unloader bracing and intra-articular injections with hyaluronic acid $(20,21)$. However, these treatments are not able to reduce the arthritic joint changes that could be observed in presence of meniscal root tears. In cases of failure of conservative treatment, the surgical option could be a choice to evaluate. However, doubts still exist concerning the proper surgical treatment regarding the age. In fact, it is obvious that in young and active patients MRR represents the best treatment choice in order to reduce pain and knee arthritis changes. In middle-aged patients (> 50 years old), meniscal root tears could be understated, creating confusion on the best treatment to choose for the patient. In this circumstance, applying three simple stitches instead of two stitches during MRR might be beneficial for healing of the posterior meniscal root, reducing the risk of meniscal failure (7). On the basis of the present study, it could be assessed that in a degenerative meniscus tissue the surgical repair applying three simple stitches could be successfully considered. In fact, the human suture-meniscal construct was able to resist to elongation during cyclic loads, remaining within the threshold of $3 \mathrm{~mm}$ of displacement.

During surgery, the meniscal root could be sutured in a different fashion, and reattached anatomically at its insertion areas using a cortical button placed at the anteromedial or anterolateral of the proximal tibia. In this phase, it is important to ensure sufficient stability of the construct, allowing an early post-operative rehabilitation program and reducing the risk of failure of the repair procedure. For this reason, different suturing methods have been proposed in order to assure a stiff fixation of the meniscal root. Furthermore, the simple suture technique (SST) trans-tibial pullout technique has been effective, combining the lowest technical difficulty and the ability to resist displacement at time zero, and comparing it to the modified Mason-Allen suture technique and the single and double double-locking loop suture technique (22). In a previous study, applying an additional suture loop was able to determine a better resistance for elongation during the fatigue tests (7). Specifically, a supplementary suture could reduce the elongation of the entire construct at 500 and 1000 cycles. Therefore, in the present study, the simple suture technique using three single loops was used and tested, considering it the most appropriate method of MRR in degenerative menisci.

The present study shows some limitations. First, this is a time zero biomechanical investigation with a very controlled laboratory situation, without effects associated with meniscal root healing that could be observed in vivo. Second, only the three single suture technique were investigated. However, other suture techniques have been described offering good and promising biomechanical results. Finally, in this study only one suture was used; therefore, varying suture types and sizes could demonstrate different results.

\section{CONCLUSION}

In this biomechanical study, it was observed that three single sutures techniques for meniscal root repair appear to be biomechanically effective for both human and porcine menisci. However, a better resistance for elongation during the fatigue tests was observed in the porcine meniscal model. This information should prove to be helpful for surgeons, to better understand the biomechanical effects of a posterior meniscal root repair.

\section{Conflict of Interest}

The authors declare that they have no conflict of interests regarding the publication of this paper.

\section{Funding}

There is no funding source.

\section{Ethical approval}

This article does not contain any studies with human participants or animals performed by any of the authors. 


\section{REFERENCES}

1. Griffith CJ, LaPrade RF, Fritts HM, Morgan PM. Posterior root avulsion fracture of the medial meniscus in an adolescent female patient with surgical reattachment. Am J Sports Med 2008; 36(4):789-792.

2. Kim JH, Chung JH, Lee DH, Lee YS, Kim JR, Ryu KJ. Arthroscopic suture anchor repair versus pullout suture repair in posterior root tear of the medial meniscus: a prospective comparison study. Arthroscopy 2011; 27(12):1644-1653.

3. Allaire R, Muriuki M, Gilbertson L, Harner CD. Biomechanical consequences of a tear of the posterior root of the medial meniscus. Similar to total meniscectomy. J Bone Joint Surg Am 2008; 90(9):1922-1931.

4. LaPrade CM, Jansson KS, Dornan G, Smith SD, Wijdicks CA, LaPrade RF. Altered tibiofemoral contact mechanics due to lateral meniscus posterior horn root avulsions and radial tears can be restored with in situ pull-out suture repairs. J Bone Joint Surg Am 2014; 96(6):471-479.

5. Marzo JM, Gurske-DePerio J Effects of medial meniscus posterior horn avulsion and repair on tibiofemoral contact area and peak contact pressure with clinical implications. Am J Sports Med 2009; 37(1):124-129.

6. Schillhammer CK, Werner FW, Scuderi MG, Cannizzaro JP. Repair of lateral meniscus posterior horn detachment lesions: a biomechanical evaluation. Am J Sports Med 2012; 40(11):2604-2609.

7. Camarda L, Pitarresi G, Lauria M, Fazzari F, D'Arienzo M. Three single loops enhance the biomechanical behavior of the transtibial pull-out technique for posterior meniscal root repair. Arch Orthop Trauma Surg 2017; 137(9):1301-1306.

8. Rosslenbroich SB, Borgmann J, Herbort M, Raschke MJ, Petersen W, Zantop T. Root tear of the meniscus: biomechanical evaluation of an arthroscopic refixation technique. Arch Orthop Trauma Surg 2013; 133(1):111-115.

9. Kim SB, Ha JK, Lee SW, Kim DW, Shim JC, Kim JG, Lee MY. Medial meniscus root tear refixation: comparison of clinical, radiologic, and arthroscopic findings with medial meniscectomy. Arthroscopy 2011; 27(3):346-354.

10. LaPrade RF, Matheny LM, Moulton SG, James EW, Dean CS. Posterior Meniscal Root Repairs: Outcomes of an Anatomic Transtibial Pull-Out Technique. Am J Sports Med 2017; 45(4):884-891.

11. Camarda L, Pitarresi G, Fazzari F, Tumino D, D’Arienzo M. Biomechanical comparison between the modified rolling-hitch and the modified finger-trap suture techniques. Arch Orthop Trauma Surg 2016; 136(11):1595-1600.

12. Kim YM, Joo YB, Noh CK, Park IY. The Optimal Suture Site for the Repair of Posterior Horn Root Tears: Biomechanical Evaluation of Pullout Strength in Porcine Menisci. Knee Surg Relat Res 2016; 28(2):147-152.

13. Cerminara AJ, LaPrade CM, Smith SD, Ellman MB, Wijdicks CA, LaPrade RF. Biomechanical evaluation of a transtibial pull-out meniscal root repair: challenging the bungee effect. Am J Sports Med 2014; 42(12):2988-2995.

14. Stärke C, Kopf S, Gröbel K, Becker R. The effect of a nonanatomic repair of the meniscal horn attachment on meniscal tension: a biomechanical study. Arthroscopy 2010; 26(3):358365.

15. Padulo J, Oliva F, Frizziero A, Maffulli N. Muscles, Ligaments and Tendons Journal. Basic principles and recommendations in clinical and field science research: 2016 update. Muscles Ligaments Tendons J 2016; 6:1-5.

16. Marchiori G, Bologna E, Lopomo N, Camarda L, Zaffagnini $\mathrm{S}$, Zingales M. Fractional order hereditariness of knee human ligament and tendon. Paper presented at VII Annual Meeting of The Italian Chapter of the European Society of Biomechanics (2017) (ESB-ITA), September 28-29 2017, Roma, Italy.

17. Deseri L, Pollaci P, Zingales M, Dayal K. Fractional hereditariness of lipid membranes: Instabilities and linearized evolution. J Mech Behav Biomed Mater 2016; 58:11-27.

18. Deseri L, Di Paola M, Zingales M, Pollaci P. Power-law hereditariness of hierarchical fractal bones. Int J Numer Method Biomed Eng 2013; 29(12):1338-1360.

19. Provenzano PP, Lakes RS, Corr DT, Vanderby R,Jr (2002) Application of nonlinear viscoelastic models to describe ligament behavior. Biomech Model Mechanobiol 2013; 1(1):45-57.

20. Bhatia S, LaPrade CM, Ellman MB, LaPrade RF. Meniscal root tears: significance, diagnosis, and treatment. Am J Sports Med 2014; 42(12):3016-3030.

21. Di Sante L., Venditto T., Ioppolo F., Paoloni M., Mangone M., Alviti F. Ultrasound guided injection of a painful knee osteoarthritis with medial meniscus extrusion: a case series study. Muscles Ligaments Tendons J 2017; 7(2):331-337.

22. LaPrade RF, LaPrade CM, Ellman MB, Turnbull TL, Cerminara AJ, Wijdicks CA. Cyclic displacement after meniscal root repair fixation: a human biomechanical evaluation. Am J Sports Med 2015; 43(4):892-898. 